\title{
A maternal high-fat, high-sucrose diet alters insulin sensitivity and expression of insulin signalling and lipid metabolism genes and proteins in male rat offspring: effect of folic acid supplementation
}

\author{
Candace E. Cuthbert ${ }^{1}$, Jerome E. Foster ${ }^{1}$ and D. Dan Ramdath ${ }^{2 *}$ \\ ${ }^{1}$ Department of Pre-Clinical Sciences, Faculty of Medical Sciences, The University of the West Indies, St. Augustine, \\ Trinidad and Tobago, West Indies \\ ${ }^{2}$ Guelph Research and Development Centre, Agriculture and Agri-Food Canada, 93 Stone Road West, Guelph, \\ ON N1G 5C9, Canada
}

(Submitted 1 February 2017 - Final revision received 31 July 2017 - Accepted 24 August 2017)

\section{Abstract}

A maternal high-fat, high-sucrose (HFS) diet alters offspring glucose and lipid homoeostasis through unknown mechanisms and may be modulated by folic acid. We investigated the effect of a maternal HFS diet on glucose homoeostasis, expression of genes and proteins associated with insulin signalling and lipid metabolism and the effect of prenatal folic acid supplementation (HFS/F) in male rat offspring. Pregnant Sprague-Dawley rats were randomly fed control (CON), HFS or HFS/F diets. Offspring were weaned on CON; at postnatal day 70 , fasting plasma insulin and glucose and liver and skeletal muscle gene and protein expression were measured. Treatment effects were assessed by one-way ANOVA. Maternal HFS diet induced higher fasting glucose in offspring $v$. HFS/F $(P=0.027)$ and down-regulation $(P<0.05)$ of genes coding for v-Akt murine thymoma viral oncogene homolog 2, resistin and v-Raf-1 murine leukaemia viral oncogene homolog 1 (Raf1) in offspring skeletal muscle and acetyl-CoA carboxylase (Acaca), fatty acid synthase and phosphatidylinositol-4,5-biphosphate 3-kinase, catalytic subunit $\beta$ in offspring liver. Skeletal muscle neuropeptide Y and hepatic Kruppel-like factor 10 were up-regulated in HFS $v$. CON offspring $(P<0 \cdot 05)$. Compared with CON, Acaca and Raf1 protein expression levels were significantly lower in HFS offspring. Maternal HFS induced higher homoeostasis model of assessment index of insulin resistance $v$. CON $(P=0.030)$ and HFS/F was associated with higher insulin $(P=0.016)$ and lower glucose $(P=0.025)$. Maternal HFS diet alters offspring insulin sensitivity and de novo hepatic lipogenesis via altered gene and protein expression, which appears to be potentiated by folate supplementation.

Key words: Fetal programming: High-fat high-sucrose diet: Folic acid: Insulin resistance: Lipid metabolism

Epidemiological studies suggest that deleterious prenatal nutrient environments can lead to an increased risk of chronic disease, including type 2 diabetes (T2D), in adult offspring ${ }^{(1,2)}$. These observations have been reconciled into the developmental origins of health and disease (DOHaD) hypothesis, which proposes that an individual faces elevated chronic disease risk in adult life when the maternal nutrient environment is incongruent with the postnatal environment ${ }^{(3)}$. Fetal overnutrition, along with maternal hyperglycaemia and hyperlipidaemia, has been linked to both obesity and T2D in adult offspring ${ }^{(4)}$. However, the mechanisms by which maternal diets effect these persistent metabolic changes are still poorly understood.

Increased consumption of energy-dense, Western diets ${ }^{(5)}$, consisting of high levels of refined carbohydrates and lipids, has been linked to obesity and $\mathrm{T} 2 \mathrm{D}^{(6-10)}$. Consequently, high-fat, high-sucrose (HFS) diets, simulating a Western diet, when fed to rodent models result in increased body weight and abdominal fat deposition, hyperinsulinaemia, and hyperglycaemia ${ }^{(11)}$. Moreover, HFS exposure in utero has been shown to alter glucose, insulin and lipid homoeostasis ${ }^{(12-14)}$ and induces adipose accumulation and fatty liver in adult rodent offspring ${ }^{(13,15)}$. The precise mechanism by which gestational HFS exposure alters glucose and insulin homoeostasis and the expression of genes and proteins associated with insulin signalling and lipid metabolism remains largely unknown. However, maternal folic acid supplementation has been shown to modulate inheritance of the deleterious metabolic effects in offspring ${ }^{(16,17)}$ and prevents the onset of hypertension, endothelial dysfunction and adiposity ${ }^{(15,18-20)}$.

Abbreviations: Acaca, acetyl-CoA carboxylase; Akt2, V-Akt murine thymoma viral oncogene homolog 2; CON, control; Ct, threshold cycle; Fasn, fatty acid synthase; HFS, high-fat/high-sucrose; HFS/F, folic-acid-supplemented, high-fat/high-sucrose; HOMA-IR, homoeostasis model of assessment index of insulin resistance; IR, insulin resistance; Klf1O, Kruppel-like factor 10; Pik3cb, phosphatidylinositol-4,5-bisphosphate 3-kinase, catalytic subunit $\beta$; PVDF, polyvinylidenedifluoride; T2D, type 2 diabetes; Raf1, V-Raf-1 murine leukaemia viral oncogene homolog 1 ; Slc2a4, solute carrier family 2, facilitated GLUT, member 4 . 
In the case of insulin sensitivity, there is conflicting evidence on the potential of gestational folic acid supplementation to influence this phenotype: prenatal exposure to high folate appears to induce greater insulin resistance (IR) in offspring $^{(21,22)}$, whereas a low-folate maternal diet induces higher body weights and adiposity, IR and high blood pressure in male offspring ${ }^{(23)}$. As such, further work is required to better understand the role of folic acid in glucose homoeostasis and its effect on the inheritance of genes and proteins associated with insulin signalling and lipid metabolism.

$\mathrm{IR}$, a precursor to $\mathrm{T} 2 \mathrm{D}$, is characterised by a decreased response to the effects of insulin on the liver and skeletal muscle, which respectively play central roles in glucose and lipid metabolism and insulin-mediated glucose uptake ${ }^{(24)}$. The HFS diet has been observed to alter insulin signalling by down-regulation of insulin receptor substrate 2 (Irs2) and v-Akt murine thymoma viral oncogene homolog $2(A k t 2)$, and to alter lipid metabolism by up-regulation of fatty acid synthase (Fasn) in the livers of mice ${ }^{(25)}$ and down-regulation of $P P A R G$ gene expression in the livers of male rats ${ }^{(26)}$. HFS feeding significantly reduces insulin-stimulated glucose transport by reducing translocation of the solute carrier family 2 , facilitates GLUT member 4 (Slc2a4) in the skeletal muscle and elevates serum insulin and TAG levels ${ }^{(27,28)}$. The lipogenic genes acetylCoA carboxylase (Acaca) and Fasn can be trans-activated by the synergistic actions of insulin and glucose ${ }^{(29)}$ and downregulated by PUFA ${ }^{(30,31)}$, so it is likely that an HFS diet may alter the regulation of these genes. Therefore, investigations of the expression of these genes and proteins associated with impaired insulin signalling and dyslipidaemia may provide useful insights into the mechanism(s) by which a prenatal HFS diet may influence offspring insulin sensitivity and adiposity.

We hypothesised that an HFS diet fed during pregnancy will increase body weights, induce IR and down-regulate insulin signalling and lipid metabolism genes and proteins in the offspring, and that folic acid supplementation of the maternal pregnancy diet will modulate these effects.

\section{Methods}

\section{Animal husbandry and experimental diets}

All animal protocols were conducted in accordance with animal husbandry standards established by the Ethics Committee, Faculty of Medical Sciences, The University of the West Indies, Trinidad and Tobago. In brief, a total of fifteen pairs of nulliparous female and male Sprague-Dawley rats, weighing 200-300g, were obtained from the Animal House facility, Department of Veterinary Medicine, and were housed individually with exposure to a $12 \mathrm{~h}$ light $-12 \mathrm{~h}$ dark cycle at $23-25^{\circ} \mathrm{C}$ throughout the experiment. Female rats were mated monogamously and the date of conception noted. After confirmation of pregnancy, females were allocated to one of three dietary feeding groups ( $n$ 5/dietary group) from conception date until parturition. The date of parturition for each dam was designated postnatal day 0 for the litter. Food and water were accessed ad libitum. The three experimental diets were prepared commercially (Dyets Inc.) and comprised AIN-93G purified rodent control diet (CON: 16\% protein,
Table 1. Diet compositions fed to Sprague-Dawley rat dams during pregnancy

\begin{tabular}{|c|c|c|c|}
\hline & \multicolumn{3}{|c|}{ Rodent diet } \\
\hline & $\mathrm{CON}$ & HFS & $\mathrm{HFS} / \mathrm{F}$ \\
\hline Sucrose $(\mathrm{g} / \mathrm{kg})$ & $100 \cdot 0$ & 452.0 & 449.0 \\
\hline Dyetrose $^{\circledR}(\mathrm{g} / \mathrm{kg})^{\star}$ & $132 \cdot 0$ & 0.0 & 0.0 \\
\hline Soyabean oil $(\mathrm{g} / \mathrm{kg})$ & $70 \cdot 0$ & $70 \cdot 0$ & $70 \cdot 0$ \\
\hline Lard $(\mathrm{g} / \mathrm{kg})$ & 0.0 & $130 \cdot 0$ & $130 \cdot 0$ \\
\hline Casein $(\mathrm{g} / \mathrm{kg})$ & $200 \cdot 0$ & $200 \cdot 0$ & $200 \cdot 0$ \\
\hline L-Cystine $(\mathrm{g} / \mathrm{kg})$ & 3.0 & 3.0 & 3.0 \\
\hline Maize starch $(\mathrm{g} / \mathrm{kg})$ & 397.5 & 47.5 & 47.5 \\
\hline$t$-Butylhydroquinone $(\mathrm{g} / \mathrm{kg})$ & 0.014 & 0.014 & 0.014 \\
\hline Cellulose (g/kg) & $50 \cdot 0$ & $50 \cdot 0$ & 50.0 \\
\hline Mineral mix $(\mathrm{g} / \mathrm{kg}) \dagger$ & $35 \cdot 0$ & $35 \cdot 0$ & $35 \cdot 0$ \\
\hline Vitamin mix $(\mathrm{g} / \mathrm{kg}) \ddagger$ & $10 \cdot 0$ & $10 \cdot 0$ & $10 \cdot 0$ \\
\hline Folic acid (mg/kg) & $2 \cdot 0$ & $2 \cdot 0$ & $5 \cdot 0$ \\
\hline Choline bitartrate $(\mathrm{g} / \mathrm{kg})$ & 2.5 & $2 \cdot 5$ & 2.5 \\
\hline Total energy (kJ/kg) & 15731.8 & $19162 \cdot 7$ & $19162 \cdot 7$ \\
\hline
\end{tabular}

CON, control; HFS, high-fat/high-sucrose; HFS/F, folic-acid-supplemented, high-fat/ high-sucrose

* Dyetrose ${ }^{\circledR}$ (Dyets), a dextrinised maize starch composed of $90-94 \%$ tetrasaccharides. † AIN-93 mineral mix (Dyet no. 210025)

$\ddagger$ AIN-93-VX vitamin mix (Dyet no. 310025). All diets provided by Dyets Inc.

$17 \%$ soyabean oil, $11 \%$ sucrose and $2 \mathrm{mg} / \mathrm{kg}$ folic acid $)^{(32)}$; HFS diet (16\% protein, $14 \%$ soyabean oil and $26 \%$ lard, $40 \%$ sucrose and $2 \mathrm{mg} / \mathrm{kg}$ folic acid) and folic-acid-supplemented HFS diet (HFS/F: $16 \%$ protein, 14\% soyabean oil and 26\% from lard, $40 \%$ sucrose and $5 \mathrm{mg} / \mathrm{kg}$ folic acid). Folic acid concentrations of 2 and $5 \mathrm{mg} / \mathrm{kg}$ of rodent diet have been used extensively in supplementation investigations ${ }^{(16-18,21,33)}$ given that these levels are analogous to the recommended daily allowance of folic acid for adults $^{(34)}$ and expectant women ${ }^{(35)}$, respectively. The complete compositions of the diets are summarised in Table 1.

After parturition, all dams were fed the CON diet throughout the lactation period. All litters were standardised to six pups per litter within $48 \mathrm{~h}$ to avoid any inequities in energy consumption among and within the dietary groups during lactation. Birth weight measurements were not taken to avoid maternal cannibalisation of the pups ${ }^{(36)}$. Litter weights were measured weekly for 10 weeks after postnatal day 7. Male offspring were weaned at postnatal day 28 and maintained on the CON diet, ad libitum. At postnatal day 70 , all animals were euthanised by administration of a lethal dose of sodium pentobarbital, and dissected along the ventral midline from the urogenital area to the sternum to expose the organs. Whole blood was collected in EDTA tubes by cardiac puncture and centrifuged to obtain plasma, which was stored at $-20^{\circ} \mathrm{C}$. The livers and skeletal muscle (gastrocnemius from hind leg) of the male offspring were collected rapidly and flashfrozen for storage at $-80^{\circ} \mathrm{C}$. Only the tissues of the male offspring were used for these analyses to avoid confounding effects of hormonal changes in the female offspring during their life cycle, which may affect weight gain and development ${ }^{(37)}$.

\section{RNA extraction and gene expression assays}

RNA was extracted from male offspring liver and skeletal muscle tissues using the RNeasy Mini kit, according to the manufacturer's instructions (Qiagen). Purified RNA was converted to complementary DNA by use of the RT2 first-strand kit 
according to the manufacturer's instructions (Qiagen) and was stored overnight at $-20^{\circ} \mathrm{C}$. Gene expression was determined using the RT2 Profiler PCR Array for the rat insulin signalling pathway (Qiagen) on an ABI 7500 real-time PCR machine (Applied Biosystems). The preset dissociation stage for the ABI 7500 real-time PCR machine was included on the thermal profile as recommended by the manufacturers (Qiagen). The threshold cycle $\left(C_{t}\right)$ values were obtained from the real-time PCR and used to calculate fold changes for the genes of interest.

\section{Western blotting}

Frozen liver and skeletal muscle tissue were homogenised with ice-cold RIPA lysis and extraction buffer (Thermo Scientific ${ }^{\mathrm{TM}}$ ) supplemented with Halt ${ }^{\mathrm{TM}}$ Protease and Phosphatase Inhibitor (Thermo Scientific ${ }^{\mathrm{TM}}$ ) using the Tissuelyser II (Qiagen) and supernatants were obtained by centrifugation at $15000 \mathrm{rpm}$ at $4^{\circ} \mathrm{C}$. Total protein concentration was determined by the Bradford protein-dye binding assay ${ }^{(38)}$ using the Pierce $^{\mathrm{TM}}$ pre-diluted protein assay Bovine Serum Albumin standards (Thermo Scientific $\left.{ }^{\mathrm{TM}}\right)$. Protein samples $(10 \mu \mathrm{g})$ were electrophoresed on 4-12\% SDS-PAGE mini-gels along with an internal protein control, and transferred onto Immobilon ${ }^{\text {TM}}{ }_{-} \mathrm{P}$ polyvinylidenedifluoride (PVDF) membranes (Sigma-Aldrich). Non-specific antibody binding was blocked by incubating the PVDF in Tris-buffered saline (TBS) containing $0.05 \%$ Tween 20 (TBST) containing either 5\% bovine serum albumin or $5 \%$ skimmed milk, according to recommendations on the primary antibody. The PVDF membranes were subsequently probed with primary antibodies specific for Acaca, Akt2, B-Raf (1:1000 dilution; Cell Signaling Technology), Slc2a4 (1:1000 dilution; Santa Cruz Biotechnology Inc.) and their phosphorylated forms. Glyceraldehyde 3-phosphate dehydrogenase (GAPDH) primary antibody (1:1000 dilution; Cell Signaling Technology) was used as a housekeeping protein control. Unbound primary antibody was removed by washing the PVDF three consecutive times in TBST. The PVDF was then probed with alkaline-phosphatase-conjugated anti-mouse IgG secondary antibody (1:1000 dilution; Cell Signaling Technology). Unbound secondary antibody was removed by washing the PVDF in TBST five times. The blots were developed using Alkaline Phosphatase conjugate substrate kit (Bio-Rad) and the blot images were captured with the GeneSnap image acquisition software (Syngene) and analysed using the ImageJ densitometric software (US National Institutes of Health).

\section{Fasting plasma glucose and insulin assays}

Fasting plasma glucose concentrations were measured using the 'Semi-micro' protocol of the Glucose liquicolor kit as per the manufacturer's instructions (Human Diagnostics Worldwide). Fasting plasma insulin concentrations were determined using the Rat/Mouse Insulin ELISA ninety-six-well plate assay kit according to the manufacturer's instructions (EMD Millipore Corporation). Insulin values were obtained by extrapolation from plotting a graph of absorbance at $450 \mathrm{~nm}$, less than that obtained at $590 \mathrm{~nm}$, on the $y$-axis against the concentration of rat insulin standards on the $\mathrm{x}$-axis. The homoeostasis model assessment index of IR (HOMA-IR), quantitative insulin check index (QUICKI) and fasting plasma glucose:insulin ratio (FGIR) were calculated as described by Cacho et al. ${ }^{(39)}$.

\section{Data analysis}

A priori and post hoc power analyses were performed using G*Power 3.1 .5 software $^{(40)}$. With an $\alpha$ level of 0.05 , power established at $80 \%$ and an effect size of 0.9 , the required total sample size was 18 . The hypothesised effect size of 0.9 was calculated from the descriptive statistics of a previous study ${ }^{(41)}$. Post hoc calculations using the hypothesised effect size and the total sample size of 15 (i.e. $n$ 5/group) indicated that the actual power achieved in this study was $79 \%$.

Data are presented as means with their standard errors. Gene expression fold change was calculated as $2^{-\Delta \Delta C_{t}}$, where $\Delta C_{t}$ for each gene is the $C_{t}$ of the housekeeping gene, $\beta$-actin, subtracted from the $C_{t}$ of the gene of interest; $\Delta\left(\Delta C_{t}\right)$ is the $\Delta C_{t}$ for the control samples subtracted from the $\Delta C_{t}$ of either the HFS or $\mathrm{HFS} / \mathrm{F}$ samples. To analyse the protein expression, the area under the densitometric graphs (AUC), corresponding to the blot intensity for each sample, was obtained from the ImageJ software. The relative density for each protein sample was determined by dividing the AUC for each sample by the AUC for the internal protein control used in each Western blot assay. The adjusted relative density for each protein of interest was normalised by dividing the relative density for the protein of interest by the relative density of the housekeeping protein GAPDH. Mean differences for the gene and protein expression data were assessed by one-way ANOVA. Bonferroni's post hoc test was conducted for multiple comparisons. For all tests, $P<0.05$ was determined to be statistically significant. All statistical analyses were performed using SPSS software for Windows version 16.0 (IBM).

\section{Results}

\section{Offspring litter weights}

There was no significant difference in food consumption patterns among the dams of the various study groups. Mean litter weights of CON and HFS/F offspring were significantly lower $(P \leq 0 \cdot 05)$ than the HFS offspring from postnatal week 7 onwards to 10 (Fig. 1).

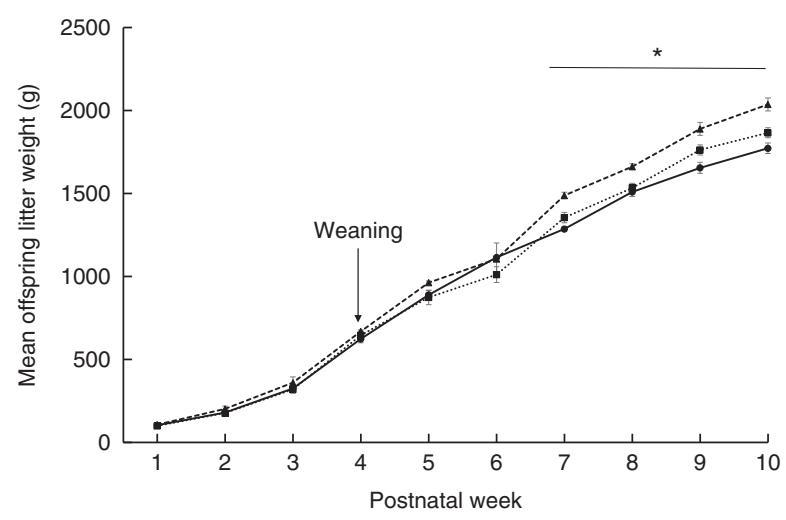

Fig. 1. Weekly mean litter weights $(\mathrm{g})$ for the control (—), high-fat, highsucrose $(--\ldots-\cdot)$ and folic-acid-supplemented high-fat high-sucrose $(\cdots-\cdots)$ offspring. * Mean litter weight values were significantly different $(P \leq 0.05)$ among the dietary groups from postnatal weeks 7 to 10 . 


\section{Liver and skeletal muscle gene expression}

There were significant treatment effects in offspring liver gene expression for four genes: Acaca, Fasn, Kruppel-like factor 10 (Klf1O) and phosphatidylinositol-4,5-biphosphate 3-kinase, catalytic subunit $\beta$ (Pik3cb) (Table 2). Hepatic Acaca (95\% CI $0.0188,0.318)$ and Pik3cb (95\% CI 0.332, 2.47) were significantly down-regulated in HFS $v$. CON. Conversely, Klf10 (95\% CI 0.0019, 0.0287) expression was significantly up-regulated in HFS $v$. CON offspring. However, hepatic Fasn

Table 2. Liver and skeletal muscle gene-fold changes among offspring exposed to prenatal high-fat/high-sucrose (HFS) and folic-acidsupplemented high-fat/high-sucrose (HFS/F) compared with the control (CON) diet

\begin{tabular}{lllll}
\hline & & \multicolumn{3}{c}{$\begin{array}{c}\text { Gene expression fold change } \\
\text { compared with CON }\end{array}$} \\
\cline { 3 - 4 } Tissues & Gene & HFS & HFS/F & $P$ \\
\hline Liver & Acaca & 0.272 & 0.332 & $0.015^{*}$ \\
& Fasn & 0.133 & 0.324 & $0.006^{\star *}$ \\
& KIf10 & 1.240 & 0.809 & $0.012^{*}$ \\
Skeletal muscle & Pik3cb & 0.130 & 0.604 & $0.011^{*}$ \\
& Akt2 & 0.559 & 0.684 & $0.038^{*}$ \\
& Cbl & 0.720 & 1.140 & $0.014^{*}$ \\
& Dok3 & 0.447 & 0.785 & $0.040^{*}$ \\
& Npy & 1.900 & 1.080 & $0.019^{*}$ \\
& Raf1 & 0.444 & 0.702 & $0.016^{*}$ \\
& Retn & 0.233 & 0.659 & $0.011^{*}$ \\
& Slc2a4 & 0.543 & 0.849 & $0.002^{* *}$ \\
& & &
\end{tabular}

Acaca, acetyl-CoA carboxylase; Fasn, fatty acid synthase; KIf10, Kruppel-like factor 10; Pik3cb, phosphatidylinositol-4,5-bisphosphate 3-kinase, catalytic subunit $\beta$; Akt2, V-Akt murine thymoma viral oncogene homolog 2; Cbl, Cbl proto-oncogene, E3 ubiquitin protein ligase; Dok3, docking protein 3; Npy, neuropeptide Y; Raf1, V-Raf-1 murine leukaemia viral oncogene homolog 1; Retn, resistin; SIc2a4, solute carrier family 2, facilitated GLUT, member 4.

Gene expression fold changes were significantly different from those of the control group: ${ }^{*} P<0.05,{ }^{* *} P<0.01$.
(95\% CI $0.252, \quad 1.33)$ gene expression was significantly down-regulated in HFS compared with the HFS/F offspring.

Offspring skeletal muscle gene expression differed significantly $(P<0 \cdot 05)$ among the study groups (Table 2$)$. In particular, Akt2 (95\% CI 0.0004, 0.0285), docking protein 3 (Dok3; 95\% CI 0.0002, 0.0087), V-Raf-1 murine leukaemia viral oncogene homolog 1 (Raf1) (95\% CI 0.0045, 0.0413), Slc2a4 (95\% CI 0.0067, 0.0276) and resistin (Retn) expression levels (95\% CI 0.0004, 0.0027) were significantly $(P<0.05)$ down-regulated in the HFS $v$. CON group, respectively. In contrast, neuropeptide Y (Npy) expression (95\% CI 0.0215, 0.489) was significantly $(P<0.05)$ up-regulated in the HFS $v$. CON group with an almost 2-fold up-regulation. $\mathrm{Cbl}$ proto-oncogene, E3 ubiquitin protein ligase (Cbl), expression (95\% CI 0.0033, 0.0300) was significantly $(P<0.05)$ down-regulated in HFS compared with HFS/F.

\section{Liver and skeletal muscle protein expression}

Liver and skeletal muscle protein expression values are shown in Table 3. Compared with CON, liver Acaca expression was significantly reduced with both HFS $(P<0.05)$ and HFS/F $(P<0.05)$ diets. Further, folic acid supplementation of the maternal HFS diet was unable to prevent the reduction in hepatic Acaca expression as significant differences were not observed between the HFS and HFS/F groups $(P>0 \cdot 05)$. In skeletal muscle, maternal HFS diet, but not supplemental folic acid, resulted in a significant reduction in phosphorylated C-Raf expression $(P<0 \cdot 05)$.

\section{Measures of glucose homoeostasis}

Table 4 shows that the offspring from the maternal HFS group had significantly higher fasting plasma glucose concentrations than their HFS/F counterparts $(P=0.025)$, indicating that the

Table 3. Liver and skeletal muscle protein expression relative to glyceraldehyde 3-phosphate dehydrogenase (GAPDH) (loading control protein) among offspring exposed to maternal control (CON), high-fat/high-sucrose (HFS) and folic-acid-supplemented high-fat/high-sucrose (HFS/F) diets (Mean values with their standard errors)

\begin{tabular}{|c|c|c|c|c|c|c|c|c|}
\hline \multirow[b]{3}{*}{ Tissues } & \multirow[b]{3}{*}{ Protein } & \multicolumn{6}{|c|}{ Offspring mean protein expression $(n 5)$} & \multirow[b]{3}{*}{$P$} \\
\hline & & \multicolumn{2}{|c|}{ CON } & \multicolumn{2}{|c|}{ HFS } & \multicolumn{2}{|c|}{$\mathrm{HFS} / \mathrm{F}$} & \\
\hline & & Mean & SEM & Mean & SEM & Mean & SEM & \\
\hline \multirow[t]{6}{*}{ Liver } & Acaca & 1.414 & 0.230 & 0.660 & $0 \cdot 108$ & 0.699 & 0.127 & $0.011^{*} \dagger$ \\
\hline & pAcaca & 0.620 & 0.123 & 3.617 & $2 \cdot 103$ & 0.680 & 0.336 & 0.222 \\
\hline & Akt2 & 0.190 & 0.056 & 0.533 & 0.171 & 0.460 & 0.142 & 0.222 \\
\hline & pAkt2 & 0.472 & 0.083 & 0.904 & 0.113 & 0.746 & 0.265 & 0.320 \\
\hline & B-Raf & 0.303 & 0.101 & 0.309 & 0.169 & 0.715 & 0.150 & 0.121 \\
\hline & pC-Raf & 3.683 & 1.718 & $21 \cdot 12$ & 11.26 & $2 \cdot 146$ & 0.493 & 0.118 \\
\hline \multirow[t]{8}{*}{ Skeletal muscle } & Acaca & 0.891 & 0.300 & 0.571 & 0.121 & 0.653 & 0.193 & 0.555 \\
\hline & pAcaca & 3.087 & 1.299 & $1 \cdot 156$ & 0.517 & 0.864 & 0.139 & 0.252 \\
\hline & Akt2 & 5.500 & $2 \cdot 160$ & $2 \cdot 178$ & 0.971 & 1.226 & 0.573 & 0.170 \\
\hline & pAkt2 & 3.622 & 1.060 & 3.580 & 1.238 & $2 \cdot 411$ & 0.541 & 0.630 \\
\hline & B-Raf & 0.972 & 0.536 & 0.382 & 0.067 & 0.851 & 0.457 & 0.488 \\
\hline & pC-Raf & $2 \cdot 596$ & 0.533 & 0.871 & 0.207 & 1.033 & 0.363 & $0.023^{*}$ \\
\hline & Slc2a4 & 4.696 & 1.475 & 2.859 & 0.281 & $2 \cdot 416$ & 0.216 & 0.271 \\
\hline & pSlc2a4 & $4 \cdot 311$ & 1.889 & 1.741 & 0.573 & $1 \cdot 123$ & 0.404 & 0.164 \\
\hline
\end{tabular}

Acaca, acetyl-CoA carboxylase; pAcaca, phosphorylated acetyl-CoA carboxylase; Akt2, V-Akt murine thymoma viral oncogene homolog 2; pAkt2, phosphorylated V-Akt murine thymoma viral oncogene homolog 2; B-Raf, B-Raf proto-oncogene, serine/threonine kinase; pC-Raf, phosphorylated C-Raf proto-oncogene, serine/threonine kinase; Slc2a4, solute carrier family 2, facilitated GLUT, member 4; pSIc2a4, phosphorylated solute carrier family 2, facilitated GLUT, member 4.

* Mean protein expression levels were significantly different between the control and HFS $(P<0.05)$.

† Mean protein expression levels were significantly different between the control and HFS/F groups $(P<0.05)$ 
Table 4. Measures of glucose homoeostasis in male offspring (Mean values with their standard errors)

\begin{tabular}{|c|c|c|c|c|c|c|c|}
\hline \multirow[b]{3}{*}{ Variables } & \multicolumn{5}{|c|}{ Offspring prenatal exposure group $(n 5)$} & & \multirow[b]{3}{*}{$P($ ANOVA $)$} \\
\hline & \multicolumn{2}{|c|}{$\mathrm{CON}$} & \multicolumn{2}{|c|}{ HFS } & \multicolumn{2}{|c|}{$\mathrm{HFS} / \mathrm{F}$} & \\
\hline & Mean & SEM & Mean & SEM & Mean & SEM & \\
\hline Glucose concentration (mM) & $7 \cdot 1$ & 0.5 & 7.5 & 0.2 & $5 \cdot 9$ & 0.2 & $0.025^{\star}$ \\
\hline Insulin concentration $(\mathrm{pmol} / \mathrm{l})$ & $286 \cdot 1$ & 46.5 & 438.2 & 81.9 & 554.9 & $15 \cdot 3$ & $0.016^{*}$ \\
\hline HOMA-IR & 2.08 & 0.26 & 3.43 & 0.55 & 3.54 & 0.21 & $0.030^{*}$ \\
\hline QUICKI & 0.271 & 0.004 & 0.257 & 0.005 & 0.254 & 0.002 & $0.018^{*}$ \\
\hline FGIR $\left(\mathrm{mg} / 10^{-4} \mathrm{U}\right)$ & 3.44 & 0.61 & 2.54 & 0.55 & 1.34 & 0.04 & $0.028^{*}$ \\
\hline
\end{tabular}

CON, control; HFS, high-fat/high-sucrose; HFS/F, folic-acid-supplemented, high-fat/high-sucrose; HOMA-IR, homoeostasis model of assessment index; QUICKI, quantitative insulin sensitivity check index; FGIR, fasting plasma glucose:insulin ratio.

* Mean glucose and insulin concentrations, HOMA-IR, QUICKI and FGIR were significantly different from those of the control group $(P<0.05)$.

folic acid supplementation of the maternal diet prevented offspring hyperglycaemia. However, fasting plasma insulin levels were significantly higher in the HFS/F than in the CON offspring $(P=0 \cdot 016)$ (Table 4). Among the study groups, HOMA-IR was lowest in CON offspring, whereas HFS/F offspring had the highest values (Table 4 ), and although there was an overall treatment effect on HOMA-IR $(P=0 \cdot 030)$, post hoc analysis failed to identify any significant group difference. CON offspring had significantly higher QUICKI values than the HFS/F offspring $(P=0 \cdot 018)$. In addition, CON offspring had a significantly $(P<0.05)$ higher FGIR than their HFS/F counterparts. Collectively, the results of QUICKI, FGIR and HOMA-IR all suggest that the HFS/F maternal diet induced IR in the offspring.

\section{Discussion}

Gestational HFS diet was hypothesised to adversely affect metabolic pathways associated with T2D, insulin signalling and lipid metabolism through trans-generational effects on glucose metabolism, gene and protein expression in the offspring. Further, folic acid supplementation was also hypothesised to augment these effects via transcriptional regulatory pathways. Our results show that prenatal exposure to HFS followed by post-weaning CON diet predisposed male offspring to significant hyperglycaemia, impaired de novo lipogenesis and higher weight gain as compared with offspring exposed to CON during gestation and post-weaning: these are all findings that support the DOHaD mismatch paradigm ${ }^{(42)}$.

Altered glucose metabolism in HFS offspring has been previously demonstrated with prenatal feeding of high-fat sucrose diets in rodents ${ }^{(11,12)}$. Similarly, a trend of higher glucose and insulin levels has been observed after gestational and lactation HFS feeding of adult male Sprague-Dawley rats ${ }^{(43)}$. Our results provide further insights into the mechanism by which gestational HFS exposure induces hyperglycaemia in offspring and suggest that this may be a result of modulations in skeletal muscle gene expression compared with CON via down-regulation of Akt2 and Raf1. Akt2 phosphorylates Raf1, which activates the downstream kinases Mek and Erk resulting in Slc2a 4 translocation to the muscle cell membrane and uptake of glucose ${ }^{(44,45)}$. Hence, down-regulation of skeletal muscle
Akt2 and Raf1 may impair insulin-induced translocation of Slc2a4, which was confirmed by down-regulation of Slc2a4 gene expression. Akt2-deficient mice had been previously observed to display impaired glucose homoeostasis in both liver and skeletal muscle ${ }^{(46)}$, whereas increased Akt protein expression in skeletal muscle of adult offspring exposed to a prenatal obesogenic diet improved their insulin sensitivity, although such a result was unexpected ${ }^{(47)}$. Our results show that expression of the phosphorylated form of Raf1 protein was significantly lower in the skeletal muscle of HFS $v$. CON offspring, which implies that Raf1 protein activity is suppressed and can less efficiently activate effector kinases that promote insulin-mediated glucose uptake. Further, our findings indicate that prenatal folic acid- supplementation reduces hyperglycaemia induced by gestational HFS diet. Taken together, these findings could account for the HFS offspring having significantly higher fasting plasma glucose concentrations than HFS/F offspring and non-significantly higher fasting plasma glucose concentrations than CON offspring.

Our results also show that HFS offspring were predisposed to significantly higher weight gain as compared with CON offspring. Higher weight gain and adiposity have been observed in male Wistar rat offspring fed HFS during pregnancy and lactation - an effect that was prevented by maternal methyl-donor supplementation with a mixture of choline, betaine, folic acid and vitamin $\mathrm{B}_{12}{ }^{(15,48)}$. Our study shows that similar preventative effects may be achieved by supplementation of maternal HFS with $3 \mathrm{mg} / \mathrm{kg}$ folic acid. Down-regulation of Retn, the hormone that suppresses the ability of insulin to stimulate glucose uptake and storage as fat ${ }^{(49)}$, suggests that the excess glucose that is not being transported into the HFS offspring skeletal muscle may instead be shunted into adipose tissue. In addition, increased body mass may also be as a result of up-regulation of $N p y$ in HFS offspring given that increased Npy secretion in HFS-fed mice has been shown to induce hyperinsulinaemia, glucose intolerance and increased visceral fat mass ${ }^{(50)}$.

The results of the hepatic gene and protein expression suggest impairments in de novo lipogenesis through decreased longchain fatty acid synthesis via down-regulation of Acaca and Fasn in HFS offspring compared with CON and HFS/F. This result was unexpected given that high-fat, high-carbohydrate diets have been shown to stimulate hepatic de novo lipogenesis ${ }^{(51)}$. 
Hyperglycaemia increases hepatic de novo lipogenesis by stimulating the release of insulin, which positively controls the lipogenic transcription factor, sterol regulatory element-binding protein 1c (SREBP-1c) ${ }^{(52)}$. SREBP-1c inturn regulates fatty acid synthesis by inducing the expression of lipogenic genes such as Acaca and Fasn ${ }^{(53)}$. However, hepatic SREBP-1C is inhibited by PUFA, which triggers the degradation of SREBP-1C mRNA ${ }^{(54)}$. Hence, in the presence of increased metabolic flux of fatty acids associated with HFS, it seems reasonable that there would be a down-regulation of fatty acid synthesis as evidenced by down-regulation of Acaca and Fasn. Interestingly, it appears that supplemental folic acid (HFS/F) was able to augment this effect to some extent.

Klf1O is a transcriptional regulator of energy metabolism, which has been shown to be induced in the liver by glucose stimulation of carbohydrate response element-binding protein (ChREBP) in a rodent model ${ }^{(55)}$. Therefore, the hyperglycaemia experienced by HFS offspring may account for the up-regulation of $\mathrm{Klf1O}$, given that it has been shown that overexpression of Klf1O inhibits glucose-stimulated ChREBP target genes such as Fasn and Acaca in rat primary hepatocytes $^{(49)}$. It is likely that up-regulation of Klf1O expression may also contribute to the inhibition of long-chain fatty acid synthesis in HFS offspring. Transcription of the Fasn promoter is stimulated by insulin via the PI3-K signalling pathway ${ }^{(56)}$; as such, hyperinsulinaemia may be the result of increased Fasn gene expression as found in the HFS/F offspring. Unphosphorylated Acaca protein expression was also significantly lower in hepatic tissue of HFS and HFS/F compared with CON offspring, which is likely to be associated with impaired fatty acid synthesis. Unlike the gene expression data, there was no significant difference in unphosphorylated Acaca protein expression between HFS and HFS/F offspring, indicating that prenatal folic acid supplementation was unable to improve fatty acid synthesis. Although the gene expression levels were significantly different among the treatment groups, the minimal effects of the HFS diet on protein expression may be due to varying levels of translation of the genes expressed. Therefore, the gene expression levels for $A k t 2, p A k t 2, \operatorname{Slc} 2 a 4$ and $p S l c 2 a 4$ may not have been high enough to produce significant differences at the level of translation to protein.

The observed changes in HOMA-IR, FGIR and QUICKI indices suggest that gestational HFS/F induced significant perturbations in glucose homoeostasis. Interestingly, folic acid supplementation decreased blood glucose, but was associated with an increase in insulin. As such, HOMA-IR was significantly lower in CON offspring than HFS and HFS/F, with no difference between the latter groups, indicating that supplemental folic acid had no protective effect on IR. We suggest that increased circulating insulin in HFS/F offspring, despite their low circulating glucose, could be attributed to a reduction in dietary folic acid as the animals transitioned between the prenatal HFS/F diet and the postnatal CON diet. It is possible that in utero the animals might have become programmed to increased folate requirements and an unsupplemented postnatal CON diet was associated with the observed impaired HOMA-IR. Unfortunately, we did not measure plasma folate levels, but folate depletion has been associated with increased hepatic lipid peroxidation products, an indicator of increased hepatic oxidative stress ${ }^{(57)}$. Further, decreased folic acid is associated with altered homocysteine metabolism, given that 5-methyl tetrahydrofolate remethylates homocysteine to methionine, and resulting hyperhomocysteinemia has consistently been associated with hyperinsulinaemia and $\mathrm{IR}^{(58)}$. Alternatively, the level of folic acid supplementation may not have been high enough to confer a protective effect against IR. A previous study demonstrated a trend of HFS offspring becoming insulin resistant, although the treatment effects were not significantly different ${ }^{(43)}$. Folic acid has been shown to improve insulin sensitivity in patients with metabolic syndrome ${ }^{(59)}$ and overweight adults ${ }^{(60)}$; both attributed to an anti-inflammatory effect of folic acid ${ }^{(60,61)}$. However, women in Pune, India, who consumed $500 \mu \mathrm{g}$ of folic acid/d during pregnancy, and had the highest erythrocyte folate levels, had 6-year-old children with IR and increased total fat mass ${ }^{(22)}$ in comparison with controls. In another study by Huang et $a l^{(21)}$, exposure to high folic acid concentrations ( $40 \mathrm{mg}$ folic acid/kg diet) in utero predisposed male rat offspring to impaired glucose tolerance and greater IR than their control and low folic acid ( $5 \mathrm{mg}$ folic acid/ $\mathrm{kg}$ diet) counterparts after 8 weeks of high-fat-diet feeding. Our findings suggest that prenatal exposure to $5 \mathrm{mg}$ of folic acid/ $\mathrm{kg}$ diet results in significantly higher insulin even in the absence of postnatal high-fat feeding. This raises concern about the effect of folic acid on IR given that prolonged hyperinsulinaemia in HFS/F offspring could result in constant exposure to stimulatory insulin concentrations and thereby increases in insulin receptor internalisation and degradation, which could lead to a reduction in the number of insulin receptors on the cell surface and eventually $\mathrm{IR}^{(62)}$. As mentioned above, there is need for further studies to define the optimal dose of folic acid needed to augment the diabetogenic effects of prenatal HFS exposure.

Hyperinsulinaemia observed in HFS/F offspring compared with their CON counterparts may be attributed to supplemental folic acid inducing a compensatory over-secretion of insulin in response to hyperglycaemia caused by the HFS diet. Higher insulin concentrations would allow increased glucose uptake by inducing the translocation of glucose transporters; this was evident in the significantly lower fasting plasma glucose concentrations in the HFS/F $v$. HFS offspring. It is possible that the effect may be mediated by the $\mathrm{Cbl}$ protein, which targets tyrosine-phosphorylated substrates, such as Irs1 and Irs2, for proteasome degradation ${ }^{(63,64)}$. As Irs1 and Irs2 phosphorylate downstream kinases of the insulin signalling pathway resulting in glucose uptake in the skeletal muscle, it was expected that significant up-regulation of $C b l$ could lead to hyperglycaemia. However, blood glucose concentrations of HFS/F offspring were significantly lower than HFS. This paradox may be explained, in part, by the compensatory mechanisms of other genes that facilitate enhanced glucose uptake. The HFS gestational diet also seems to predispose the offspring hepatocytes to decreased downstream insulin signalling, as suggested by down-regulation of $P i k 3 c b^{(65)}$, the catalytic $p 110 \beta$ subunit of PI3-kinase, which is involved in signal transduction of insulin. Furthermore, Pik3cb is a positive regulator of insulin secretion and therefore the significantly lower $P i k 3 c b$ gene expression in the HFS $v$. CON offspring may account for the lower insulin 
secretion in the HFS offspring ${ }^{(66)}$. It is therefore possible that suppression of insulin secretion may also account for the hyperglycaemia observed in the HFS offspring.

Another possible contributor to the onset of IR in the offspring is high dietary fructose (derived from the sucrose) content of the HFS diet. Consumption of high-fructose diets has been associated with induction of IR, impaired glucose tolerance, hypertriacylglycerolaemia and hyperinsulinaemia in animal models ${ }^{(67,68)}$ and impaired insulin sensitivity in humans ${ }^{(69)}$. Fructose metabolism and hyperglycaemia can result in oxidative damage through the production of reactive oxygen species $^{(70)}$, and it has been suggested that this oxidative stress might play a contributory role in the aetiology of IR and eventually T2D.

It is likely that the differences in gene and protein expression observed among the CON, HFS and HFS/F offspring may occur through epigenetic mechanisms. Epigenetics refers to a heritable change in gene expression that occurs without DNA sequence changes and has been proposed to be the mechanism that links environmental influences, such as maternal diet, to the aetiology of $\mathrm{T} 2 \mathrm{D}^{(71,72)}$. The most widely studied mechanism is DNA methylation, which involves the reversible transfer of methyl groups from dietary folic acid to cytosine molecules of $\mathrm{DNA}^{(72)}$. Maternal nutrition and supplements to maternal diets, such as folic acid, may influence the DNA methylation of the offspring ${ }^{(73,74)}$. Hence, the observed alterations to the hepatic fat metabolism and skeletal muscle glucose uptake in HFS offspring and IR in the HFS/F offspring may lie in the DNA methylation of genes related to insulin secretion.

\section{Conclusions}

The results of this study provide compelling evidence for the DOHaD theory of mismatch. Our investigation has shown that prenatal exposure to HFS diet significantly alters hepatic fat metabolism and skeletal muscle glucose uptake in male offspring, at both transcription and translation. In addition, supplementation of the maternal HFS diet with $5 \mathrm{mg}$ of folic acid $/ \mathrm{kg}$ diet significantly increases circulating insulin and reduces plasma glucose levels. The HFS diet predisposes offspring to IR as compared with the CON diet. The results of this study can potentially have an impact on health policy for T2D by highlighting the role of maternal nutrition in the prevention of adverse metabolic effects related to impaired insulin signalling and fat metabolism in the offspring. In addition, results from this study will help to further examine the underlying mechanisms of the developmental origins of adult health and disease.

\section{Acknowledgements}

The authors thank Dr Shamjeet Singh of the Pre-Clinical Sciences Department, Dr Jenelle Johnson of the School of Veterinary Medicine and Ms Aileen Hawke of the Guelph Research and Development Centre, Agriculture and Agri-Food Canada, for advice and technical services rendered.

This work was supported by a grant from the Caribbean Public Health Agency and Agriculture \& Agri-Food Canada
Project no. 1343; C. E. C was the recipient of a CARICOMCanada Emerging Leaders Scholarship.

D. D. R., J. E. F. and C. E. C. conceived and designed the study, interpreted the data and drafted the manuscript. C. E. C. conducted the research and acquired the data and performed the statistical analysis. All authors read and approved the final manuscript.

The authors declare that there are no conflicts of interest.

\section{References}

1. Godfrey KM \& Barker DJ (2000) Fetal nutrition and adult disease. Am J Clin Nutr 71, 1344S-1352S.

2. Godfrey KM \& Barker DJ (2001) Fetal programming and adult health. Public Health Nutr 4, 611-624.

3. Wadhwa PD, Buss C, Entringer S, et al. (2009) Developmental origins of health and disease: brief history of the approach and current focus on epigenetic mechanisms. Semin Reprod Med 27, 358-368

4. Dabelea D (2007) The predisposition to obesity and diabetes in offspring of diabetic mothers. Diabetes Care 30, Suppl. 2, S169-S174.

5. Cordain L, Eaton SB, Sebastian A, et al. (2005) Origins and evolution of the Western diet: health implications for the 21st century. Am J Clin Nutr 81, 341-354.

6. Bray GA \& Popkin BM (1998) Dietary fat intake does affect obesity! Am J Clin Nutr 68, 1157-1173.

7. Swinburn BA, Caterson I, Seidell JC, et al. (2004) Diet, nutrition and the prevention of excess weight gain and obesity. Public Health Nutr 7, 123-146.

8. McAuley KA, Hopkins CM, Smith KJ, et al. (2005) Comparison of high-fat and high-protein diets with a high-carbohydrate diet in insulin-resistant obese women. Diabetologia 48, 8-16.

9. van Dam RM, Rimm EB, Willett WC, et al. (2002) Dietary patterns and risk for type 2 diabetes mellitus in U.S. men. Ann Intern Med 136, 201-209.

10. Hu FB (2011) Globalization of diabetes: the role of diet, lifestyle, and genes. Diabetes Care 34, 1249-1257.

11. Murase T, Mizuno T, Omachi T, et al. (2001) Dietary diacylglycerol suppresses high fat and high sucrose dietinduced body fat accumulation in C57BL/6J mice. J Lipid Res 42, 372-378.

12. Zheng J, Xiao X, Zhang Q, et al. (2015) Maternal and postweaning high-fat, high-sucrose diet modulates glucose homeostasis and hypothalamic POMC promoter methylation in mouse offspring. Metab Brain Dis 30, 1129-1137.

13. Cordero P, Gomez-Uriz AM, Milagro FI, et al. (2012) Maternal weight gain induced by an obesogenic diet affects adipose accumulation, liver weight, and insulin homeostasis in the rat offspring depending on the sex. J Endocrinol Invest 35, 981-986.

14. Wanjihia VW, Ohminami H, Taketani $\mathrm{Y}$, et al. (2013) Induction of the hepatic stearoyl-CoA desaturase 1 gene in offspring after isocaloric administration of high fat sucrose diet during gestation. J Clin Biochem Nutr 53, 150-157.

15. Cordero P, Milagro FI, Campion J, et al. (2014) Supplementation with methyl donors during lactation to high-fat-sucrose-fed dams protects offspring against liver fat accumulation when consuming an obesogenic diet. J Dev Orig Health Dis 5, 385-395.

16. Lillycrop KA, Phillips ES, Jackson AA, et al. (2005) Dietary protein restriction of pregnant rats induces and folic acid supplementation prevents epigenetic modification of hepatic gene expression in the offspring. J Nutr 135, 1382-1386. 
17. Burdge GC, Lillycrop KA, Phillips ES, et al. (2009) Folic acid supplementation during the juvenile-pubertal period in rats modifies the phenotype and epigenotype induced by prenatal nutrition. J Nutr 139, 1054-1060.

18. Torrens C, Brawley L, Anthony FW, et al. (2006) Folate supplementation during pregnancy improves offspring cardiovascular dysfunction induced by protein restriction. Hypertension 47, 982-987.

19. Qipshidze N, Metreveli N, Lominadze D, et al. (2011) Folic acid improves acetylcholine-induced vasoconstriction of coronary vessels isolated from hyperhomocysteinemic mice: an implication to coronary vasospasm. I Cell Physiol 226, 2712-2720.

20. Seto SW, Lam TY, Or PMY, et al. (2010) Folic acid consumption reduces resistin level and restores blunted acetylcholine-induced aortic relaxation in obese/diabetic mice. J Nutr Biochem 21, 872-880.

21. Huang Y, He Y, Sun X, et al. (2014) Maternal high folic acid supplement promotes glucose intolerance and insulin resistance in male mouse offspring fed a high-fat diet. Int J Mol Sci 15, 6298-6313.

22. Yajnik CS, Deshpande SS, Jackson AA, et al. (2008) Vitamin $\mathrm{B}_{12}$ and folate concentrations during pregnancy and insulin resistance in the offspring: the Pune Maternal Nutrition Study. Diabetologia 51, 29-38.

23. Sinclair KD, Allegrucci C, Singh R, et al. (2007) DNA methylation, insulin resistance, and blood pressure in offspring determined by maternal periconceptional $\mathrm{B}$ vitamin and methionine status. Proc Nat Acad Sci 104, 19351-19356.

24. Stumvoll M \& Gerich J (2001) Clinical features of insulin resistance and beta cell dysfunction and the relationship to type 2 diabetes. Clin Lab Med 21, 31-51.

25. Yang Z-H, Miyahara H, Takeo J, et al. (2012) Diet high in fat and sucrose induces rapid onset of obesity-related metabolic syndrome partly through rapid response of genes involved in lipogenesis, insulin signalling and inflammation in mice. Diabetol Metab Syndr 4, 1-10.

26. Ragab SMM, Abd Elghaffar SK, El-Metwally TH, et al. (2015) Effect of a high fat, high sucrose diet on the promotion of nonalcoholic fatty liver disease in male rats: the ameliorative role of three natural compounds. Lipids Health Dis 14, 83.

27. Youngren JF, Paik J \& Barnard RJ (2001) Impaired insulinreceptor autophosphorylation is an early defect in fat-fed, insulin-resistant rats. J Appl Physiol 91, 2240-2247.

28. Barnard RJ, Roberts CK, Varon SM, et al. (1998) Diet-induced insulin resistance precedes other aspects of the metabolic syndrome. J Appl Physiol 84, 1311-1315.

29. Stoeckman AK \& Towle HC (2002) The role of Srebp-1c in nutritional regulation of lipogenic enzyme gene expression. J Biol Chem 277, 27029-27035.

30. Sampath H \& Ntambi JM (2005) Polyunsaturated fatty acid regulation of genes of lipid metabolism. Ann Rev Nutr 25, 317-340.

31. Jump DB (2008) $n-3$ Polyunsaturated fatty acid regulation of hepatic gene transcription. Curr Opin Lipidol 19, 242-247.

32. Reeves PG, Nielsen FH \& Fahey GC (1993) AIN-93 purified diets for laboratory rodents: final report of the American Institute of Nutrition ad boc writing committee on the reformulation of the AIN-76A rodent diet. J Nutr 123, 1939-1951.

33. Hoile SP, Lillycrop KA, Grenfell LR, et al. (2012) Increasing the folic acid content of maternal or post-weaning diets induces differential changes in phosphoenolpyruvate carboxykinase mRNA expression and promoter methylation in rats. Br J Nutr 108, 852-857.

34. Institute of Medicine (1998) Dietary Reference Intakes for Thiamin, Riboflavin, Niacin, Vitamin B6, folate, Vitamin
B12, Pantothenic acid, Biotin, and Choline. Washington, DC: The National Academies Press.

35. Yates AA, Schlicker SA \& Suitor CW (1998) Dietary reference intakes: the new basis for recommendations for calcium and related nutrients, B vitamins, and choline. I Am Diet Assoc 98, 699-706.

36. Lane-Petter W (1968) Cannibalism in rats and mice. Proc R Soc Med 61, 1295-1296.

37. Figueiredo HF, Dolgas CM \& Herman JP (2002) Stress activation of cortex and hippocampus is modulated by sex and stage of estrus. Endocrinology 143, 2534-2540.

38. Bradford MM (1976) A rapid and sensitive method for the quantitation of microgram quantities of protein utilizing the principle of protein-dye binding. Anal Biochem 72, 48-54.

39. Cacho J, Sevillano J, de Castro J, et al. (2008) Validation of simple indexes to assess insulin sensitivity during pregnancy in Wistar and Sprague-Dawley rats. Am J Physiol Endocrinol Metab 295, E1269-E1276.

40. Faul F, Erdfelder E, Lang A-G, et al. (2007) G*Power 3: a flexible statistical power analysis program for the social, behavioral, and biomedical sciences. Behav Res Methods 39, 175-191.

41. Cordero P, Gomez-Uriz AM, Campion J, et al. (2013) Dietary supplementation with methyl donors reduces fatty liver and modifies the fatty acid synthase DNA methylation profile in rats fed an obesogenic diet. Genes Nutr 8, 105-113.

42. Godfrey KM, Lillycrop KA, Burdge GC, et al. (2007) Epigenetic mechanisms and the mismatch concept of the developmental origins of health and disease. Pediatr Res 61, 5 Part 2, 5R-10R.

43. Latouche C, Heywood SE, Henry SL, et al. (2014) Maternal overnutrition programs changes in the expression of skeletal muscle genes that are associated with insulin resistance and defects of oxidative phosphorylation in adult male rat offspring. J Nutr 144, 237-244.

44. Tan S-X, Ng Y \& James DE (2010) Akt inhibitors reduce glucose uptake independently of their effects on Akt. Biochem J 432, 191-198.

45. Kohn AD, Summers SA, Birnbaum MJ, et al. (1996) Expression of a constitutively active Akt Ser/Thr kinase in 3T3-L1 adipocytes stimulates glucose uptake and glucose transporter 4 translocation. J Biol Chem 271, 31372-31378.

46. Cho H, Mu J, Kim JK, et al. (2001) Insulin resistance and a diabetes mellitus-like syndrome in mice lacking the protein kinase Akt2 (PKB $\beta$ ). Science 292, 1728-1731.

47. Shelley P, Martin-Gronert MS, Rowlerson A, et al. (2009) Altered skeletal muscle insulin signaling and mitochondrial complex II-III linked activity in adult offspring of obese mice. Am J Physiol Regul Integr Comp Physiol 297, R675-R681.

48. Cordero P, Gonzalez-Muniesa P, Milagro FI, et al. (2015) Perinatal maternal feeding with an energy dense diet and/or micronutrient mixture drives offspring fat distribution depending on the sex and growth stage. J Anim Physiol Anim Nutr 99, 834-840.

49. Barnes KM \& Miner JL (2009) Role of resistin in insulin sensitivity in rodents and humans. Curr Protein Pept Sci 10, 96-107.

50. Kuo LE, Czarnecka M, Kitlinska JB, et al. (2008) Chronic stress, combined with a high-fat/high-sugar diet, shifts sympathetic signaling toward neuropeptide y and leads to obesity and the metabolic syndrome. Ann N Y Acad Sci 1148, 232-237.

51. Strable MS \& Ntambi JM (2010) Genetic control of de novo lipogenesis: role in diet-induced obesity. Crit Rev Biochem Mol Biol 45, 199-214.

52. Foretz M, Guichard C, Ferre P, et al. (1999) Sterol regulatory element binding protein-1c is a major mediator of insulin action 
on the hepatic expression of glucokinase and lipogenesisrelated genes. Proc Natl Acad Sci U S A 96, 12737-12742.

53. Yang ZH, Miyahara H, Takeo J, et al. (2012) Diet high in fat and sucrose induces rapid onset of obesity-related metabolic syndrome partly through rapid response of genes involved in lipogenesis, insulin signalling and inflammation in mice. Diabetol Metab Syndr $\mathbf{4}, 32$.

54. Duarte JA, Carvalho F, Pearson M, et al. (2014) A high-fat diet suppresses de novo lipogenesis and desaturation but not elongation and triglyceride synthesis in mice. I Lipid Res 55, 2541-2553.

55. Iizuka K, Takeda J \& Horikawa Y (2011) Krüppel-like factor-10 is directly regulated by carbohydrate response element-binding protein in rat primary hepatocytes. Biochem Biophys Res Commun 412, 638-643.

56. Sul HS, Latasa M-J, Moon Y, et al. (2000) Regulation of the fatty acid synthase promoter by insulin. J Nutr 130, 315S-320S.

57. Huang P, Hsu Y, Lin H, et al. (2001) Folate depletion and elevated plasma homocysteine promote oxidative stress in rat livers. $J$ Nutr 131, 33-38.

58. Meigs JB, Jacques PF, Selhub J, et al. (2001) Fasting plasma homocysteine levels in the insulin resistance syndrome. Diabetes Care 24, 1403-1410.

59. Setola E, Monti LD, Galluccio E, et al. (2004) Insulin resistance and endothelial function are improved after folate and vitamin $\mathrm{B}_{12}$ therapy in patients with metabolic syndrome: relationship between homocysteine levels and hyperinsulinemia. Eur J Endocrinol 151, 483-489.

60. Solini A, Santini E \& Ferrannini E (2006) Effect of short-term folic acid supplementation on insulin sensitivity and inflammatory markers in overweight subjects. Int I Obes (Lond) 30, 1197-1202.

61. Gonda TA, Kim YI, Salas MC, et al. (2012) Folic acid increases global DNA methylation and reduces inflammation to prevent helicobacter-associated gastric cancer in mice. Gastroenterology 142, 824-833.

62. Shanik MH, Xu Y, Škrha J, et al. (2008) Insulin resistance and hyperinsulinemia: is hyperinsulinemia the cart or the horse? Diabetes Care 31, Suppl. 2, S262-S268.

63. Thien CBF \& Langdon WY (2001) Cbl: many adaptations to regulate protein tyrosine kinases. Nat Rev Mol Cell Biol 2, 294-307.
64. Nakao R, Hirasaka K, Goto J, et al. (2009) Ubiquitin ligase Cbl-b Is a negative regulator for insulin-like growth factor 1 signaling during muscle atrophy caused by unloading. Mol Cell Biol 29, 4798-4811.

65. Kolic J, Spigelman AF, Plummer G, et al. (2013) Distinct and opposing roles for the phosphatidylinositol 3-OH kinase catalytic subunits $\mathrm{p} 110 \alpha$ and $\mathrm{p} 110 \beta$ in the regulation of insulin secretion from rodent and human beta cells. Diabetologia 56, 1339-1349.

66. Bieche I, Bougneres P, Dechartres A, et al. (2008) Association analysis indicates that a variant GATA-binding site in the PIK3CB promoter is a cis-acting expression quantitative trait locus for this gene and attenuates insulin resistance in obese children. Diabetes 57, 494-502.

67. Basciano H, Federico L \& Adeli K (2005) Fructose, insulin resistance, and metabolic dyslipidemia. Nutr Metab (Lond) 2, 5 .

68. Elliott SS, Keim NL, Stern JS, et al. (2002) Fructose, weight gain, and the insulin resistance syndrome. Am J Clin Nutr 76, 911-922.

69. Aeberli I, Hochuli M, Gerber PA, et al. (2013) Moderate amounts of fructose consumption impair insulin sensitivity in healthy young men. Diabetes Care 36, 150-156.

70. Busserolles J, Gueux E, Rock E, et al. (2003) Oligofructose protects against the hypertriglyceridemic and pro-oxidative effects of a high fructose diet in rats. $J$ Nutr $\mathbf{1 3 3}$, 1903-1908.

71. Ling C \& Groop L (2009) Epigenetics: a molecular link between environmental factors and type 2 diabetes. Diabetes 58, 2718-2725.

72. Seki Y, Williams L, Vuguin PM, et al. (2012) Minireview: epigenetic programming of diabetes and obesity: animal models. Endocrinology 153, 1031-1038.

73. Lillycrop KA, Slater-Jefferies JL, Hanson MA, et al. (2007) Induction of altered epigenetic regulation of the hepatic glucocorticoid receptor in the offspring of rats fed a proteinrestricted diet during pregnancy suggests that reduced DNA methyltransferase- 1 expression is involved in impaired DNA methylation and changes in histone modifications. Br J Nutr 97, 1064-1073.

74. Lillycrop KA, Rodford J, Garratt ES, et al. (2010) Maternal protein restriction with or without folic acid supplementation during pregnancy alters the hepatic transcriptome in adult male rats. Br J Nutr 103, 1711-1719. 